\title{
Cluster CMDs from N-body Simulations: Stellar and Binary Evolution on GRAPE
}

\author{
Jarrod R. Hurley \\ American Museum of Natural History, Department of Astrophysics, \\ Central Park West at 79th Street, New York, NY 10024, USA
}

\begin{abstract}
The Colour-Magnitude diagram (CMD) is an important observational tool for use in the study of star clusters. Thus it is important to include a consistent treatment of stellar and binary evolution in $N$ body codes to allow the generation and interaction of the full range of stellar populations. In this way realistic cluster models can be compared with observed cluster populations via the respective CMDs. Preliminary results of such an approach using the Aarseth NBODY4 code on the GRAPE-6 are presented here. In particular, by using a stellar evolution algorithm that includes metallicity as a free parameter, the stellar populations of clusters at various stages of evolution can be studied. Interesting formation cases directly influenced by the cluster environment are highlighted for various stellar sub-populations.
\end{abstract}

\section{Introduction}

There is by now an overwhelming body of evidence to suggest that the evolution of a star cluster can be greatly influenced by the evolution of its members, whether they be single stars or binaries (e.g., Heggie, these proceedings). Massloss from stars as they evolve off the main-sequence (MS) will cause the cluster to relax and possibly even to disperse. Mass transfer in close binaries may lead to the coalescence of the two stars which deprives the cluster of a source of fuel in its fight against core-collapse. As a final example, the formation of a black hole will greatly affect the internal dynamics of the cluster if it is retained.

Conversely, we see many examples of the cluster environment affecting the behaviour of stars (e.g., Hurley et al. 2001). This is most clearly demonstrated by viewing the CMDs of star clusters: a number of stellar populations not expected from standard stellar evolution theory are visible (Bailyn 1995). The most commonly identified of these are the blue stragglers (BSs) and the extreme blue horizontal branch (BHB) stars, or blue sub-dwarfs (sdBs). BSs lie above and blueward of the MS turn-off in a cluster CMD and the sdBs appear as a vertical extension of the HB. M30, a core-collapsed and metal-poor globular cluster (GC), exhibits both BSs and sdBs in its CMD (Guhathakurta et al. 1998). If the stellar sub-populations, as identified by the region they occupy in the CMD, are plotted as cumulative radial distributions, i.e. population gradient, then the BSs in M30 appear concentrated towards the cluster core while the bright giants are centrally depleted. Owing to the high stellar density 
of the cluster core this suggests an explanation based on dynamical interactions between the cluster stars.

CMD appearance varies from cluster to cluster. This is clearly demonstrated when looking at the morphology of the HB. Rich et al. (1997) observe four metalrich GCs with well populated red-HB components but find that only two of these have prominent BHB extensions. Furthermore, a number of clusters have a gap in their HB (e.g., Ferraro et al. 1990). BSs have been found in all GC cores that have been well studied, with as many as 200 contained in M3. However, M15 appears to have relatively less BSs than other dense clusters. Population gradients generally show that BSs are centrally concentrated but in M3 the radial distribution appears to be bi-modal (Ferraro et al. 1993) suggesting that BSs in low-density regions form from primordial binaries and those in highdensity regions from collisions between MS stars. Not all clusters show a central depletion of giants. Testa et al. (2001) find that in NGC6626 the giants are more concentrated towards the core than the HB stars.

It seems clear that observed CMDs show a dynamical signature and that interactions between stars play a major role in producing variations in CMD appearance. Differences from cluster to cluster may be related to properties of the cluster environment, such as the central density. Necessarily the interaction between the evolution of the member stars and the dynamical evolution of the cluster itself must be consistently accounted for in any theoretical model. The inclusion of algorithms that treat stellar and binary evolution in models of star clusters naturally allows the production of simulated CMDs. The possibility then exists for the stellar populations within these CMDs to be modified by dynamical interactions and therefore much can be learnt by the comparison of theory and observation using this powerful tool.

\section{Sub-population Formation Channels}

A number of possible channels exist for the formation of the various interesting stellar sub-populations observed in the CMDs of star clusters or, on the other hand, for the depletion of certain populations. In general these involve some sort of binary interaction and/or dynamical encounters between the cluster stars. A few relevant cases are highlighted here while a comprehensive review of the subject is provided by Bailyn (1995).

\subsection{Blue Stragglers}

A collision between two MS stars, either in an hyperbolic encounter or at periastron within an eccentric binary, is a popular choice for the mechanism that produces BSs. The result is a more massive MS star that is rejuvenated relative to other stars of the same mass and thus evolves to become a BS. This will also be the case when a binary evolves to a Case A mass transfer state: a MS star filling its Roche-lobe and transferring mass to its companion, a less massive MS star, followed by coalescence of the two stars as the orbit shrinks owing to angular momentum loss. These channels create an isolated BS unless produced from a binary that was itself a member of a higher-order hierarchical system. However, the possibility exists for the BS to be subsequently exchanged into a nearby binary. 
BSs in short-period binaries could be produced from Case B mass transfer where a MS star accretes material from a sub-giant or giant. The BS will be created when the mass of the MS star exceeds the MS turn-off mass of the cluster. Mass transfer will continue at a steady rate until the Roche-lobe filling star evolves to become a white dwarf (WD). Formation channels for BSs in longperiod binaries include stable mass-transfer from an asymptotic giant branch (AGB) star to a MS star, and accretion of mass from an AGB star wind by a MS star. A scenario that does not involve collisions or binary evolution is that BSs may simply be single MS stars that are experiencing some sort of interior mixing, possibly the result of rapid rotation, that extends their MS lifetime. This is not a well-supported theory (Leonard 1996).

\subsection{Blue Horizontal Branch Stars}

The sdBs are generally thought to be HB stars with unusually thin hydrogen envelopes. The more depleted the envelope is, the more the helium-burning core will shine through, and the bluer the star will appear. Any formation scenario must account for the fact that these stars are observed along a sequence in the CMD, and that HB stars of a particular age will have relatively the same core-mass. One possibility is that sdBs are the result of collisions involving a giant branch (GB) star where, depending on the impact parameter of the collision, part or all of the giants envelope is stripped. Mass transfer from a Roche-lobe filling giant to its companion could possibly be interrupted by a dynamical encounter with a third body. Instead of steadily transferring mass until it evolved to become a WD, the giant would appear as a BHB star when it eventually evolved off the GB.

Soker \& Harpaz (2000) have suggested that the distribution of stars on the HB may be determined by interactions with planets. In this case the evolved parent star accretes an orbiting planet and the angular momentum transferred to the star is enough to noticeably affect its subsequent evolution. On the other hand, the merger of two helium-WDs could create a helium-burning star with a thin hydrogen envelope, i.e. an sdB (Iben 1990). D'Antona, Vietri \& Pesce (1995) argue that 13 extreme BSs observed in M15 are actually sdBs formed in this manner.

It may simply be that giant stars do not all experience mass-loss from their surface at the same rate (D'Cruz et al. 1996). However, introducing a stellar wind that varies from star to star does appear to be a fairly contrived solution to the observations. Another possibility is that WDs formed during common-envelope evolution emerge from the common-envelope with a thin layer of material on their surface so that they actually appear as sdBs.

\subsection{Giant Depletion}

Any formation channel for sdBs that involves stripping the envelope of a giant can also explain an observed depletion of these stars. The effects of masssegregation in a star cluster should concentrate the giant population towards the central regions of the cluster but as the converse is often found to be the case it seems probable that their presence in the core leads to their destruction. The large cross-section for collision provided by giants relative to other cluster stars, coupled with the stellar density being highest in the core, makes depletion 
via collisions seem likely. However, stars spend a relatively short period of their life on the GB.

If a star is a member of a binary it is most likely to initiate a phase of mass transfer when it is on the sub-giant or GB. Binary-binary and binarysingle encounters can lead to hardening of a binaries orbit, or exchange of one component into another binary, which increases the chance of mass transfer. So giants may be depleted as a result of direct collisions or via a succession of weaker encounters that perturb the orbital properties of the cluster binaries.

\section{Tools of Investigation}

To model the fascinating process of star cluster evolution the Aarseth NBODY4 code (Aarseth 1999; Hurley et al. 2001) is used. Importantly NBODY4 incorporates all aspects of the single star evolution (SSE) package created by Hurley, Pols \& Tout (2000). This package comprises a set of functions that describe the evolution of a star from the zero-age MS up to, and including, remnant stages of evolution. It provides rapid updating of the stellar variables and includes metallicity as a free parameter. The binary star evolution (BSE) algorithm described by Hurley, Tout \& Pols (2001) is also contained within NBODY4. Features of binary evolution such as tidal circularization and synchronization of the orbit, angular momentum loss, common-envelope evolution and coalescence, in addition to Roche-lobe overflow and mass transfer by a stellar wind, are all modelled in detail. The evolution of the single and binary stars is performed in-step with integration of the dynamics.

The applicability of the $N$-body method to the study of star clusters owes much to the development of the GRAPE series of special-purpose computers (Makino, these proceedings). Findings presented in this work are primarily the result of simulations performed on a prototype GRAPE- 6 board located at the American Museum of Natural History in New York. The GRAPE-6, which has only recently become available, operates at $\sim 30$ Gflops per chip and represents a factor of 100 increase in computing power over its predecessor the GRAPE- 4 .

\section{Binary Population Synthesis}

It is feasibly possible to reach most of the populated areas of observed star cluster CMDs solely through binary interaction. By varying the initial conditions of the binary population, such as the distributions that govern the choice of orbital period and mass-ratio for each binary, it is possible to create differences in CMD appearance. However, care must be taken to ensure that these distributions remain physically plausible.

There are obvious examples where binary population synthesis fails to explain the observations. BSs found in eccentric binaries and super-BSs, those that have a mass greater than twice the cluster turn-off mass, are two of these (Hurley et al. 2001). In many cases the relative numbers of stars in various regions of the CMD, e.g. BSs to MS stars or sdBs to giants, is just not good enough from population synthesis.

The results of Hurley, Tout \& Pols (2001), using the BSE algorithm with reasonable choices for the initial parameters of the binaries, suggests that per 2000 
binaries approximately 1-2 BSs, 1 cataclysmic variable (CV), and 10 double-WD systems would form. Results from $N$-body models of open clusters (described in next section) show that these numbers change to 3-4 BSs, $1 \mathrm{CV}$, and 20 doubleWDs. An enhancement of BSs and double-WDs is clearly seen, even within the moderate density conditions of the open cluster environment. The numbers for each sub-population in the $N$-body models will be related to the relative segregation timescales for the various progenitor systems, i.e. the time spent by these systems in or near the cluster core. Considering only systems that formed from primordial binaries, double-WDs will have originated from heavier binaries than CVs, and therefore the mass-segregation timescale will be shorter for their progenitors. This leaves more scope for dynamics to influence their evolution. In the case of BSs they will generally form from two fairly massive MS stars.

\section{5. $\quad N$-body models}

In general, simulations performed so far with the 16-chip prototype GRAPE-6 board have involved 20000 stars with $10 \%$ primordial binaries. Stellar masses are distributed between the limits of $0.1-50 M_{\odot}$ according to a realistic initial mass function (see Hurley et al. 2001 for full details of typical initial conditions used with NBODY4). The velocity dispersion of the stars is $\sim 2 \mathrm{~km} \mathrm{~s}^{-1}$ and the stellar density in the core is $\sim 10^{3}$ stars $\mathrm{pc}^{-3}$, so these are strictly simulations of open clusters. Planetary systems have also been included in these models (Hurley \& Shara 2001).

\subsection{Colour-Magnitude Diagrams}

Figure 1 shows a CMD from a snapshot of a simulation with $Z=0.004$ at an age of $1336 \mathrm{Myr}$. This simulation continued until $4500 \mathrm{Myr}$ when approximately $25 \%$ of the original cluster remained bound. A full range of stellar types is clearly visible in Figure 1. There are a large number of double-WD binaries, some of which are possible type Ia supernovae candidates (see Shara, these proceedings). One CV is present, as are two binaries composed of a WD and a naked helium star component.

The four BSs present at this time are all in binaries and all formed via some interaction with the cluster environment. One is in a $1040 \mathrm{~d}$ circular orbit with a WD companion after increasing its mass from 1.62 to $1.78 M_{\odot}$ owing to mass transfer from the AGB progenitor of the WD. This occurred after a 3 -body exchange interaction created the binary. A $2.2 M_{\odot}$ BS has a sub-giant companion which is currently filling its Roche-lobe, i.e. stable Case B mass transfer, but only after perturbations to the initial orbit caused the period to decrease. The orbit is circular with a period of $0.52 \mathrm{~d}$. Two of the BSs have MS companions and long-period eccentric orbits. The first of these was originally a $1.5 M_{\odot} \mathrm{MS}$ star that was exchanged into a highly eccentric binary which was the inner component of a triple system. Perturbations drove the inner eccentricity even higher until the two MS stars collided to create the $2.3 M_{\odot}$ BS which remains bound to the third star. The second of these has a mass of $2.8 M_{\odot}$ and formed via Case A mass transfer in a primordial binary. It then underwent two exchange interactions to reach its current situation. This BS binary is the most massive system in the cluster at the time and quickly sinks to the centre 


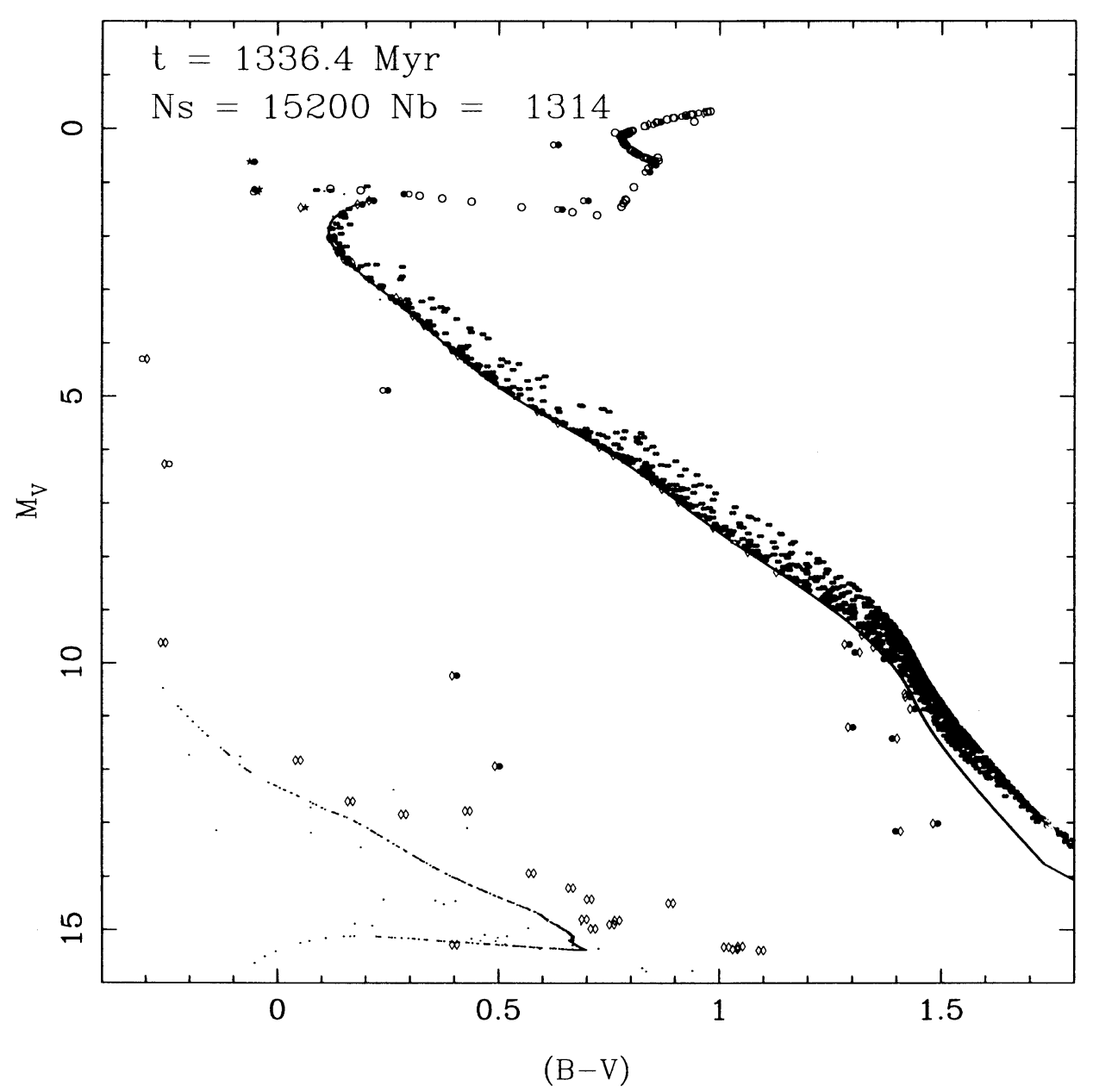

Figure 1. $\mathrm{CMD}$ for an $N$-body simulation with $Z=0.004$ at an age of $1336 \mathrm{Myr}$. There are 13013 single stars and 1314 binaries remaining at this time. Main-sequence stars (dots), blue stragglers (stars), sub-giants, giants and naked helium stars (open circles) and white dwarfs (dots) are distinguished. Binary stars are denoted by overlapping symbols appropriate to the stellar type of the components, with main-sequence binary components depicted with filled circles and white dwarf binary components as diamonds. Bolometric corrections computed by Kurucz (1992) from synthetic stellar spectra are used to convert theoretical stellar quantities to observed colours. These cc: rections are strictly not valid for WDs and extremely cool giants. The cluster turn-off mass is $1.74 M_{\odot}$ and there are $4 \mathrm{BSs}$, all of which are in binaries. There are also 18 double-WD systems and one CV. 


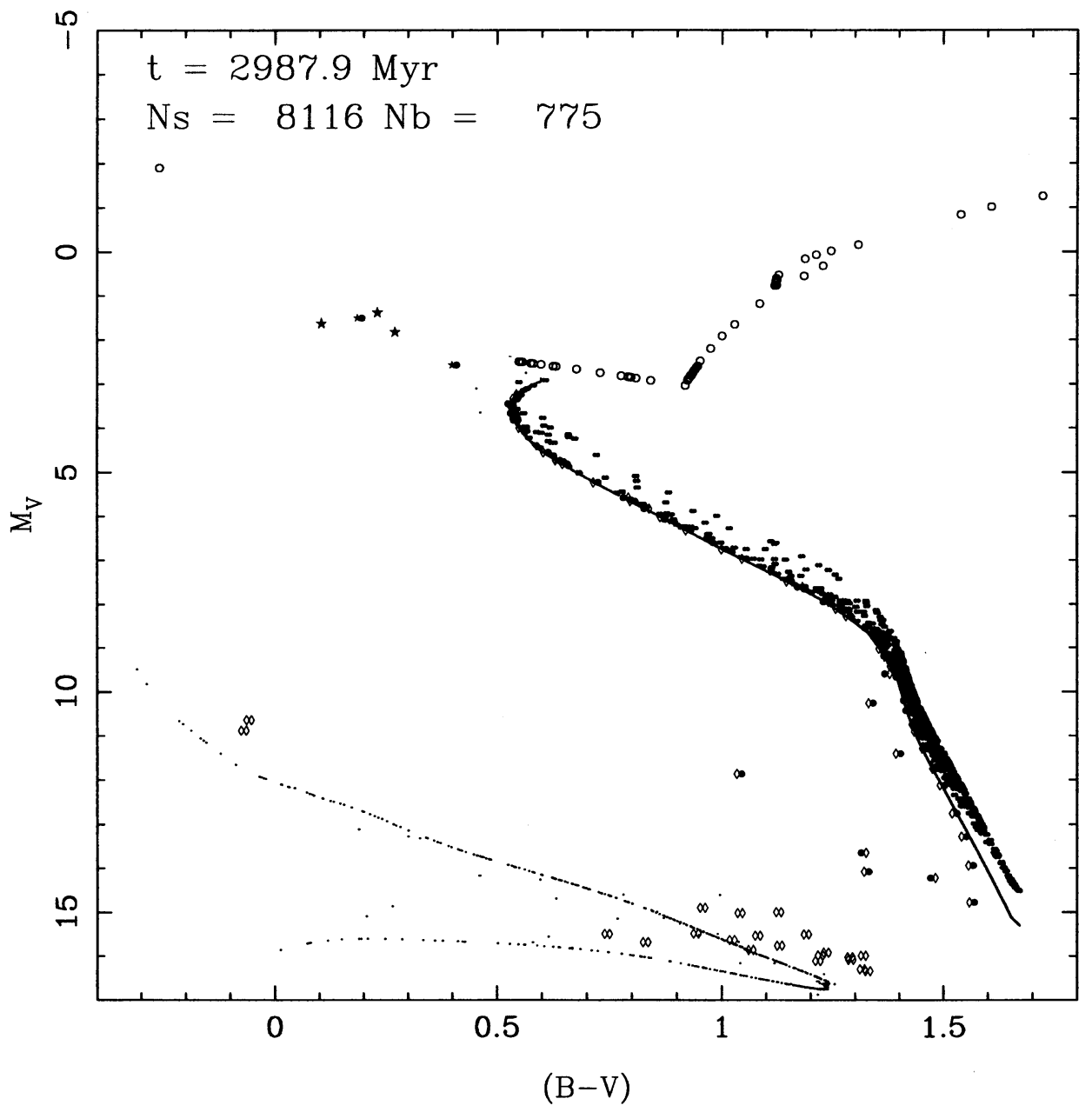

Figure 2. As Figure 1 for an $N$-body simulation with $Z=0.02$ at an age of $2987 \mathrm{Myr}$. The cluster turn-off mass is $1.46 M_{\odot}$ and there are 5 BSs, 2 of which are in eccentric binaries with MS star companions, where the binary system is the result of an exchange interaction. All BSs formed via the MS-MS coalescence path and have masses ranging from $1.53-2.01 M_{\odot}$. There are also 21 double-WD systems. An extremely blue AGB star is visible in the upper left-hand corner of the CMD. This star has only $0.01 M_{\odot}$ remaining in its envelope and is about to become a carbon-oxygen WD. It would possibly be observed as a planetary nebula. 
of the cluster. The BS is involved in further exchange interactions and after coalescing with another MS star becomes a super-BS. An even more striking example of the promiscuous nature of BSs in star clusters is described in Hurley et al. (2001). While the possibility of any particular star becoming a BS is fairly random, subsequent interactions are almost inevitable.

In the simulation highlighted in Figure 1 a BS also formed as a result of a hyperbolic collision between two MS stars. A $0.32 M_{\odot}$ star that began life in the core of the cluster slowly drifted out to the half-mass radius, owing to masssegregation, where at $1300 \mathrm{Myr}$ it collided with a $0.93 M_{\odot}$ star. The relative velocity of the two stars at impact was $2.8 \mathrm{~km} \mathrm{~s}^{-1}$ and the collision product is assumed to be fully mixed with no mass-loss. When the cluster is $3850 \mathrm{Myr}$ old this star appears on the CMD as a BS and by $4500 \mathrm{Myr}$ it has sunk inside the cluster core.

Figure 2 shows a CMD from a snapshot of a simulation with $Z=0.02$ at an age of $2987 \mathrm{Myr}$.

\subsection{Population Gradients}

Figure 3 shows a series of model population gradients. As expected the binaries, being more massive on average than the single stars, segregate inwards within the cluster. Giants also segregate towards the core of the cluster. As yet there is no evidence for a central depletion of giants and, in particular, no case of a direct collisions involving a giant has been observed in the simulations so far. This should change when the number density of stars used in the simulations is increased. Weak evidence for the depletion of giants in the core can be seen in Figure 18 of Hurley et al. (2001). BSs are primarily created in the cluster core and then remain in the core.

\section{An Interesting Case of Giant Depletion and sdB Formation}

As we utilise the capabilities of the GRAPE-6 in the near future and begin to conduct simulations more representative of GC conditions, i.e. greater particle number and higher stellar density, the statistics of stellar interactions will improve. This should lead to a larger variety of formation channels for the stellar populations of interest. In the meantime it is informative to examine some of the interesting formation scenarios that have presented themselves to date. In addition to the BS cases illustrated above one such example is given here.

A primordial binary comprised MS stars of 0.6 and $1.6 M_{\odot}$ with an eccentricity of 0.42 and an orbital period of $\sim 10^{5} \mathrm{~d}$. Residing in the core of the cluster this binary suffered a series of weak perturbations to its orbit which drove the eccentricity up to 0.95 . The $1.6 M_{\odot}$ star, now a sub-giant, was involved in two exchange interactions, finally settling into a $12000 \mathrm{~d}$ orbit with eccentricity of 0.9 about a $1.4 M_{\odot}$ MS star. Tidal forces within the binary circularize the orbit resulting in a separation of $250 R_{\odot}$. The sub-giant evolves onto the GB, fills its Roche-lobe, and common-envelope evolution begins. This strips the envelope of the giant and leaves a helium-WD and a MS star separated by $52 R_{\odot}$. The MS star subsequently evolves to the GB, fills its Roche-lobe, and another commonenvelope event ensues, resulting in a pair of 0.4 and $0.3 M_{\odot}$ helium-WDs with an 

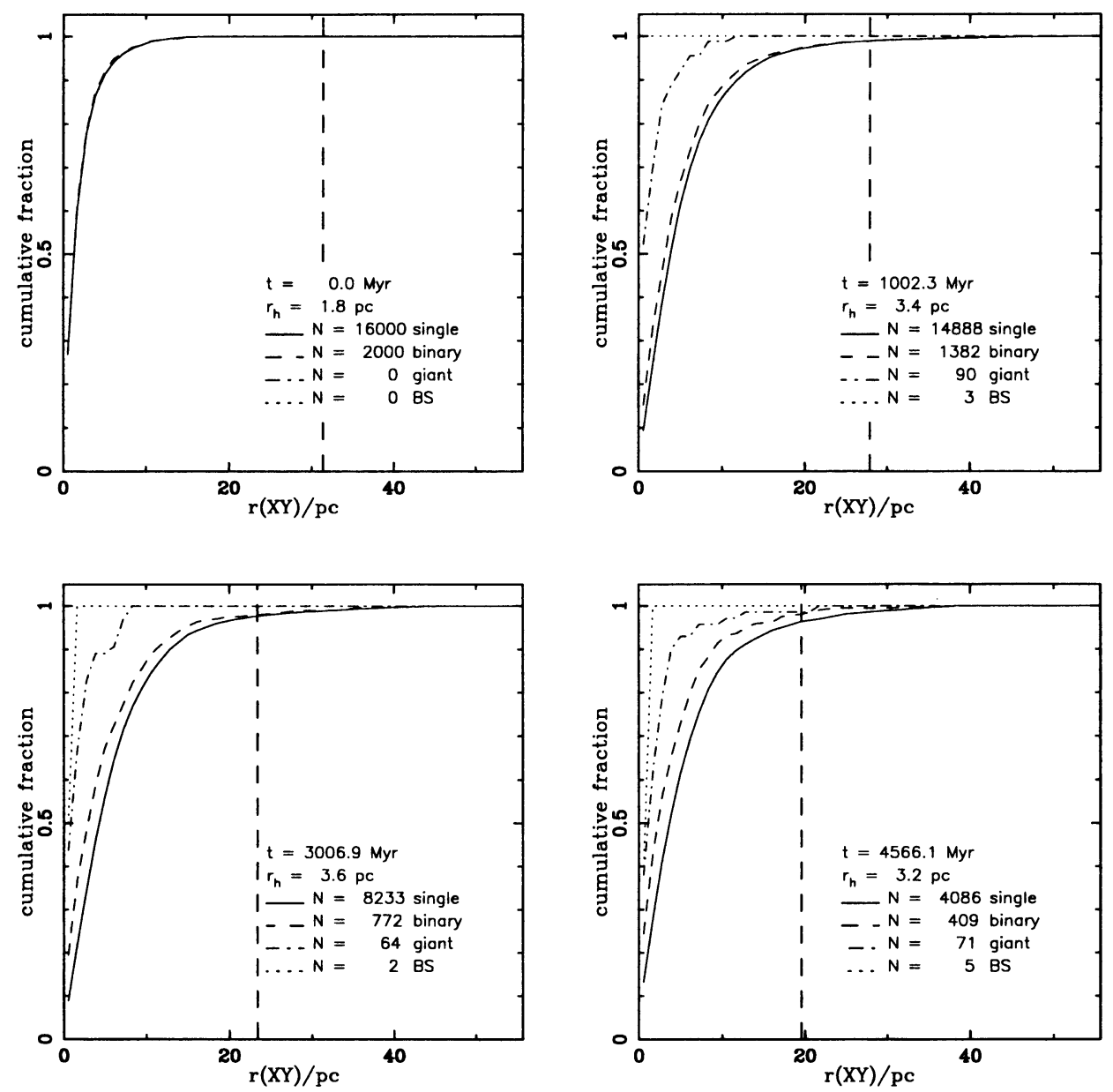

Figure 3. Cumulative radial profiles in the XY-plane for an $N$-body simulation with $Z=0.004$ at four selected times. The single star, binary, giant, and blue straggler sub-populations are identified. The tidal radius of the cluster is denoted by the vertical dashed line and the half-mass radius, $r_{\mathrm{h}}$, is also given. A standard Galactic tidal field is used. Note that stars are not actually removed from the simulation until they are at a distance greater than two tidal radii from the cluster centre. 
orbital period of $0.7 \mathrm{~d}$. Owing to gravitational radiation this system will easily merge within $10^{10} \mathrm{yr}$ to possibly form an sdB.

\section{Summary}

The introduction of the GRAPE- 6 hardware, coupled with $N$-body codes that have the capability to produce realistic cluster models, places the field of star cluster research in an extremely interesting position. An exciting aspect of this is the capability to investigate and understand the range of stellar populations that appear in cluster CMDs, and how these are affected by dynamical interactions between cluster stars.

Acknowledgments. I would like to thank Jun Makino and the University of Tokyo for the loan of a prototype GRAPE- 6 board. Thanks also to the LOC of IAU208 for providing generous financial support throughout the duration of this symposium. Immense gratitude is extended to Sverre Aarseth for his tireless work in producing state-of-the-art $N$-body codes. Without his efforts this work would not be possible. Christopher Tout, Onno Pols, Michael Shara and Piet Hut have all contributed to this work in various ways.

\section{References}

Aarseth, S.J. 1999, PASP, 111, 1333

Bailyn, C.D. 1995, ARA\&A, 33, 133

D'Antona, F., Vietri, M., \& Pesce, E. 1995, MNRAS, 272, 730

D'Cruz, N.L., Dorman, B., Rood, R.T., \& O'Connell, R.W. 1996, ApJ, 466, 359

Ferraro, F.R., Clementini, G., Fusi Pecci, F., Buonanno, R., \& Alcaino, G. 1990, A\&AS, 84, 59

Ferraro, F.R., Fusi Pecci, F., Cacciari, C., Corsi, C., Buonanno, R., Fahlman, G.G., \& Richer, H.B. 1993, AJ, 106, 2324

Guhathakurta, P., Webster, Z.T., Yanny, B., Schneider, D.P., \& Bahcall, J.N. 1998, AJ, 116, 1757

Hurley, J.R., Pols, O.R., \& Tout, C.A. 2000, MNRAS, 315, 543

Hurley, J.R., Tout, C.A., Aarseth, S.J., \& Pols, O.R. 2001, MNRAS, 323, 630

Hurley, J.R., Tout, C.A., \& Pols, O.R. 2001, MNRAS, accepted

Hurley, J.R., \& Shara, M.M. 2001, ApJ, accepted

Iben, I.Jr. 1990, ApJ, 353, 215

Kurucz, R.L. 1992, in Barbuy B., Renzini A., eds. Proc. IAU Symp. 149, The

Stellar Populations of Galaxies. (Kluwer: Dordrecht) Vol. 7, p. 225

Leonard, P.J.T. 1996, ApJ, 470, 521

Rich, R.M., et al. 1997, ApJ, 484, L25

Soker, N., \& Harpaz, A. 2000, MNRAS, 317, 861

Testa, V., Corsi, C.E., Andreuzzi, G., Iannicola, G., Marconi, G., Piersimoni, A.-M., \& Buonanno, R. 2001, AJ, 121, 916 